\title{
A Study of Some Nonlinear Partial Differential Equations by Using Adomian Decomposition Method and Variational Iteration Method
}

\author{
Marha M. Shehata \\ Department of Mathematics, Faculty of Science, Zagazig University, Zagazig, Egypt \\ Email: dr.maha 32@hotmail.com \\ Received 19 May 2015; accepted 21 June 2015; published 26 June 2015 \\ Copyright (C) 2015 by author and Scientific Research Publishing Inc. \\ This work is licensed under the Creative Commons Attribution International License (CC BY). \\ http://creativecommons.org/licenses/by/4.0/ \\ (c) (i) Open Access
}

\section{Abstract}

In this paper, a numerical solution of nonlinear partial differential equation, Benjamin-Bona-Mahony (BBM) and Cahn-Hilliard equation is presented by using Adomain Decomposition Method (ADM) and Variational Iteration Method (VIM). The results reveal that the two methods are very effective, simple and very close to the exact solution.

\section{Keywords}

Wave Variables, Adomian Decomposition Method (ADM), Variational Iteration Method (VIM), Nonlinear Partial Differential Equation PDEs, BBM and Cahn-Hilliard Equations

\section{Introduction}

In this paper, we discuss the solution of the nonlinear BBM equation [1]

$$
u_{t}=u_{x x t}-u_{x}-u u_{x}
$$

subject to the initial condition

$$
u(x, 0)=\operatorname{sech}^{2}(x / 4)
$$

The Cahn-Hilliard equation [2]:

$$
u_{t}=u_{x x}-u^{3}+u
$$


subject to the initial condition

$$
u(x, 0)=1 / 1+\mathrm{e}^{\frac{x}{\sqrt{2}}} .
$$

[3] [4] derived a variety of exact travelling wave solutions of distinct physical structures for the BBM equation, where the Tanh and the sine-cosine methods were used. Also [5] is devoted to analyzing the physical structures of the nonlinear dispersive variants of the BBM equation, where new exact solutions with compact and noncompact structures for BBM are derived. [6] applied the decomposition method to obtain explicit and numerical solutions of different types of generalized BBM. Many articles have investigated Cahn-Hilliard equation analytically and numerically, [7] applied the finite difference method to obtain the numerical solution of Cahn-Hilliard equation. [8]-[10] used the Exp-function method to obtain exact solutions of Cahn-Hilliard. [11] solved these equations by Differential Transform Method.

In the beginning of the 1980, a so-called Adomiande composition method (ADM), which appeared in [12]-[15] used (ADM) to solve coupled kdv equation.

\section{The Adomian Decomposition (ADM)}

In this section, $\mathrm{ADM}$ is explained. For this, we consider a general nonlinear partial differential equation in the following form.

$$
\begin{gathered}
L(u(x, t))+N(u(x, t))=g(x, t) \\
u(x, 0)=f(x) .
\end{gathered}
$$

where $L=\frac{\partial(\cdot)}{\partial t}$ which is assumed easily invertible, $N(u(x, t))$ presents the nonlinear term and $g$ is the source term. Appling the inverse operator $L^{-1}=\int_{0}^{t}(\cdot) \mathrm{d} s$ to both sides of (5) and using the given conditions we obtain

$$
u(x, t)=f(x)+L^{-1}(g(x, t))-L^{-1}(N(u(x, t)))
$$

using the given conditions, the ADM defines the solution $u$ by the series in the following form.

$$
u(x, t)=\sum_{n=0}^{\infty} u_{n}(x, t)
$$

and the nonlinear operator $N(u)$ presents by an infinite series of the so-called Adomian's

$$
N(u(x, t))=\sum_{n=0}^{\infty} A_{n}
$$

where $u_{n}(x, t), n \geq 0$ are the components of $u(x, t)$ that will be easy determined and $A_{n}$ are called Adomian's polynomials and defined by

$$
A_{n}\left(u_{0}, u_{1}, \cdots, u_{n}\right)=\frac{1}{n !}\left[\frac{\mathrm{d}^{n}}{\mathrm{~d} \lambda^{n}}\left(\sum_{i=0}^{\infty} \lambda^{i} u_{i}(x, t)\right)\right]_{\lambda=0}, \quad n \succ 0 .
$$

From the above considerations, the decomposition method defines the components $u_{n}(x, t)$ for $n \geq 0$, by the following recursive relationships,

$$
\begin{gathered}
u_{0}(x, t)=f(x)+L^{-1}(g(x, t)) \\
u_{n+1}(x, t)=-L_{t}^{-1}\left[A_{n}\right], \quad n \geq 0 .
\end{gathered}
$$

Finally, the approximate solution for $u(x, t)$ is obtained by truncating the series

$$
u(x, t)=\sum_{n=0}^{\infty} u_{n}(x, t)
$$


For more details about ADM and its convergence, see [12]-[14] [16]

Now, we first consider a general form of nonlinear equation

$$
P\left(u, u_{t}, u_{x}, u_{x x}, u_{x t}, u_{x x x}, u_{x x t}, \cdots\right)=0 .
$$

Second, we introduce the wave variable

$$
\zeta=\lambda(x-c t)
$$

so that

$$
u(x, t)=U(\zeta)
$$

the PDF (14) convert to an ODE

$$
P\left(U, c \lambda U^{\prime}, \lambda U^{\prime}, \lambda^{2} U^{\prime \prime}, c \lambda^{2} U^{\prime \prime}, \lambda^{3} U^{\prime \prime \prime}, c \lambda^{3} U^{\prime \prime \prime}, \cdots\right)=0 .
$$

\section{Application of ADM}

In this article, we investigate some example of the nonlinear partial differential equations

\subsection{Benjamin-Bona-Mahony}

$$
u_{t}=u_{x x t}-u_{x}-u u_{x}
$$

subject to the initial condition

$$
u(x, 0)=\operatorname{sech}^{2}(x / 4)
$$

with the exact solution is

$$
u(x, t)=\operatorname{sech}^{2}\left(\frac{x}{4}-\frac{t}{3}\right) .
$$

By using (15), Equations (18) and (19) converted to the ODE

$$
(1-c) U+c \lambda^{2} U^{\prime \prime}+\frac{1}{2} U^{2}=0,
$$

subject to the initial conditions

$$
U(0)=1, \quad U^{\prime}(0)=0 .
$$

Applying the ADM to (21)-(22), we obtain

$$
\begin{aligned}
& U^{\prime \prime}=-\frac{1}{2 c \lambda^{2}} U^{2}-\frac{(1-c)}{c \lambda^{2}} U, \\
& L(U)=-\frac{1}{2 c \lambda^{2}} U^{2}-\frac{(1-c)}{c \lambda^{2}} U,
\end{aligned}
$$

we apply the inverse operator $L^{-1}$ on both sides (24) we get

$$
\sum_{k=0}^{\infty} U_{k}(\zeta)=U(0)+U^{\prime}(0) \zeta-\frac{1}{2 c \lambda^{2}} L^{-1}\left(\sum_{k=0}^{\infty} A_{k}(\zeta)\right)+\frac{(1-c)}{c \lambda^{2}} L^{-1}\left(\sum_{k=0}^{\infty} U_{k}(\zeta),\right.
$$

where the components of $A_{k}$ are the so-called Adomian polynomials, for each $k, A_{k}$ depends on

$$
\begin{gathered}
U_{0}(\zeta), U_{1}(\zeta), \cdots, U_{k}(\zeta) \\
A_{0}=\left(U_{0}\right)^{2}, A_{1}=2 U_{0} U_{1}, A_{2}=\left(U_{1}\right)^{2}+2 U_{0} U_{2}, \cdots
\end{gathered}
$$

The components $U_{k}(\zeta)$ can be computed as 


$$
\begin{aligned}
& U_{0}(\zeta)=1 \\
& U_{1}(\zeta)=\frac{(1-c)}{c \lambda^{2}} L^{-1}\left(U_{0}\right)-\frac{1}{c \lambda^{2}} L^{-1}\left(A_{0}\right) \\
& U_{2}(\zeta)=\frac{(1-c)}{c \lambda^{2}} L^{-1}\left(U_{1}\right)-\frac{1}{c \lambda^{2}} L^{-1}\left(A_{1}\right) \\
& \ldots \\
& U_{0}(\zeta)=1 \\
& U_{1}(\zeta)=-\zeta^{2} \\
& U_{2}(\zeta)=\frac{2}{3} \zeta^{4} \\
& U_{3}(\zeta)=-\frac{1}{9} \zeta^{6} \\
& \ldots \\
& U(\zeta)=\sum_{k=0}^{4} U_{k}(\zeta)=1-\zeta^{2}+\frac{2}{3} \zeta^{4}-\frac{1}{9} \zeta^{6},
\end{aligned}
$$

Then,

$$
u(x, t)=1-(\lambda(x-c t))^{2}+\frac{2}{3}(\lambda(x-c t))^{4}-\frac{1}{9}(\lambda(x-c t))^{6}
$$

Then approximation solution of Equation (18) is $u(x, t)=u_{0}+u_{1}+u_{2}+\cdots$ with third-order approximation. Now we compare exact solution with Adomain Decomposition Method (ADM) solution in Figure 1.

\subsection{The Cahn-Hilliard Equation}

$$
u_{t}=u_{x x}-u^{3}+u
$$

subject to the initial condition

$$
u(x, 0)=\frac{1}{1+\mathrm{e}^{\frac{x}{\sqrt{2}}}},
$$
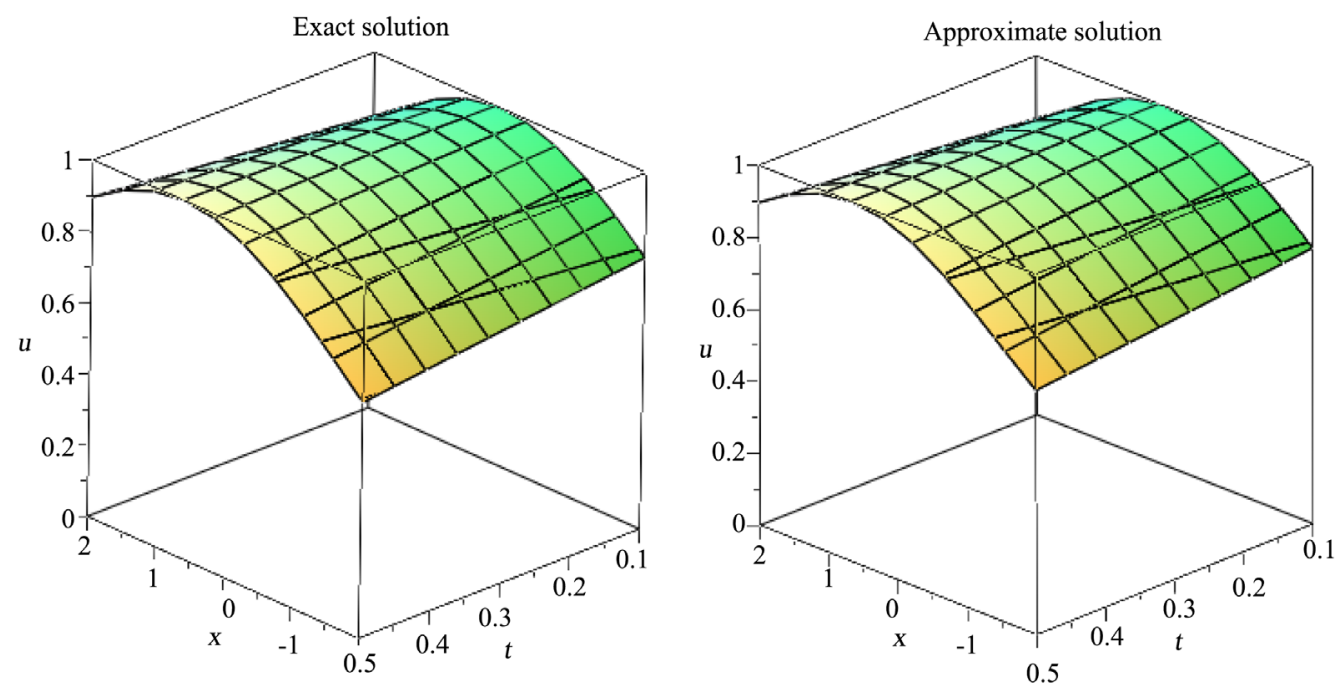

Figure 1. Exact and approximate solutions to BBM equation by ADM when $-2 \prec x \prec 2$ and $t=0.1,0.2,0.3,0.4,0.5$. 
where the exact solution is

$$
u(x, t)=\frac{1}{1+\mathrm{e}^{\frac{x}{\sqrt{2}}-\frac{3 t}{2}}} .
$$

By using (15), Equations (30) and (31) converted to the ODE

$$
-c \lambda U^{\prime}-\lambda^{2} U^{\prime \prime}+\frac{1}{2} U^{3}-U=0,
$$

subject to the initial conditions

$$
U(0)=1 / 2, \quad U^{\prime}(0)=-1 / 4 .
$$

Applying the ADM to (33)-(34), we get

$$
\begin{aligned}
U^{\prime \prime} & =-\frac{c}{\lambda} U^{\prime}+\frac{1}{2 \lambda^{2}} U^{3}-\frac{1}{\lambda^{2}} U, \\
L(U) & =-\frac{c}{\lambda} U^{\prime}+\frac{1}{2 \lambda^{2}} U^{3}-\frac{1}{\lambda^{2}} U,
\end{aligned}
$$

we apply the inverse operator $L^{-1}$ on both sides (36) we have

$$
\begin{gathered}
U=U(0)+\zeta U^{\prime}(0)-L^{-1}\left[\frac{c}{\lambda} U^{\prime}+\frac{1}{2 \lambda^{2}} U^{3}-\frac{1}{\lambda^{2}} U\right], \\
\sum_{k=0}^{\infty} U_{k}(\zeta)=-\frac{c}{\lambda} L^{-1}\left(\sum_{k=0}^{\infty}\left(U_{k}\right)^{\prime}\right)+\frac{1}{2 \lambda^{2}} L^{-1}\left(\sum_{k=0}^{\infty} A_{k}\right)-\frac{1}{\lambda^{2}} L^{-1}\left(\sum_{k=0}^{\infty} U_{k}\right) .
\end{gathered}
$$

where the components of $A_{k}$ are the so-called Adomian polynomials, for each $k, A_{k}$ depends on

$$
\begin{gathered}
U_{0}(\zeta), U_{1}(\zeta), \cdots, U_{k}(\zeta) . \\
A_{0}=\left(U_{0}\right)^{3}, A_{1}=3\left(U_{0}\right)^{2} U_{1}, A_{2}=3 U_{0}\left(U_{1}\right)^{2}+3\left(U_{0}\right)^{2} U_{2}, \cdots
\end{gathered}
$$

The components $U_{k}(\zeta)$ can be computed as

$$
\begin{gathered}
U_{0}(\zeta)=\frac{1}{2}-\frac{1}{4} \zeta \\
U_{1}(\zeta)=\frac{-c}{\lambda} L^{-1}\left(U_{0}\right)^{\prime}+\frac{1}{c \lambda^{2}} L^{-1}\left(A_{0}\right)-\frac{1}{\lambda^{2}} L^{-1}\left(U_{0}\right) \\
U_{2}(\zeta)=\frac{-c}{\lambda} L^{-1}\left(U_{1}\right)^{\prime}+\frac{1}{c \lambda^{2}} L^{-1}\left(A_{1}\right)-\frac{1}{\lambda^{2}} L^{-1}\left(U_{1}\right) \\
\ldots \\
U(\zeta)=\sum_{k=0}^{4} U_{k}(\zeta)=\frac{1}{2}-\frac{1}{4} \zeta-\frac{1}{16} \zeta^{2}+\frac{5}{96} \zeta^{3}+\frac{3}{128} \zeta^{4}-\frac{1}{1280} \zeta^{5} \\
u(x, t)=\frac{1}{2}-\frac{1}{4}(\lambda(x-c t))-\frac{1}{16}(\lambda(x-c t))^{2}+\frac{5}{96}(\lambda(x-c t))^{3}+\frac{3}{128}(\lambda(x-c t))^{4}-\frac{1}{1280}(\lambda(x-c t))^{5}
\end{gathered}
$$

Then approximation solution of Equation (30) is $u(x, t)=u_{0}+u_{1}+\cdots$. Now we compare exact solution with Adomain Decomposition Method (ADM) solution in Figure 2.

\section{Variational Iteration Method (VIM) [17]}

Let consider the differential equation

$$
L u+N u=f(t)
$$

where $L$ and $N$ are linear and nonlinear operators, respectively, and $f(t)$ is the inhomogeneous term. In the 

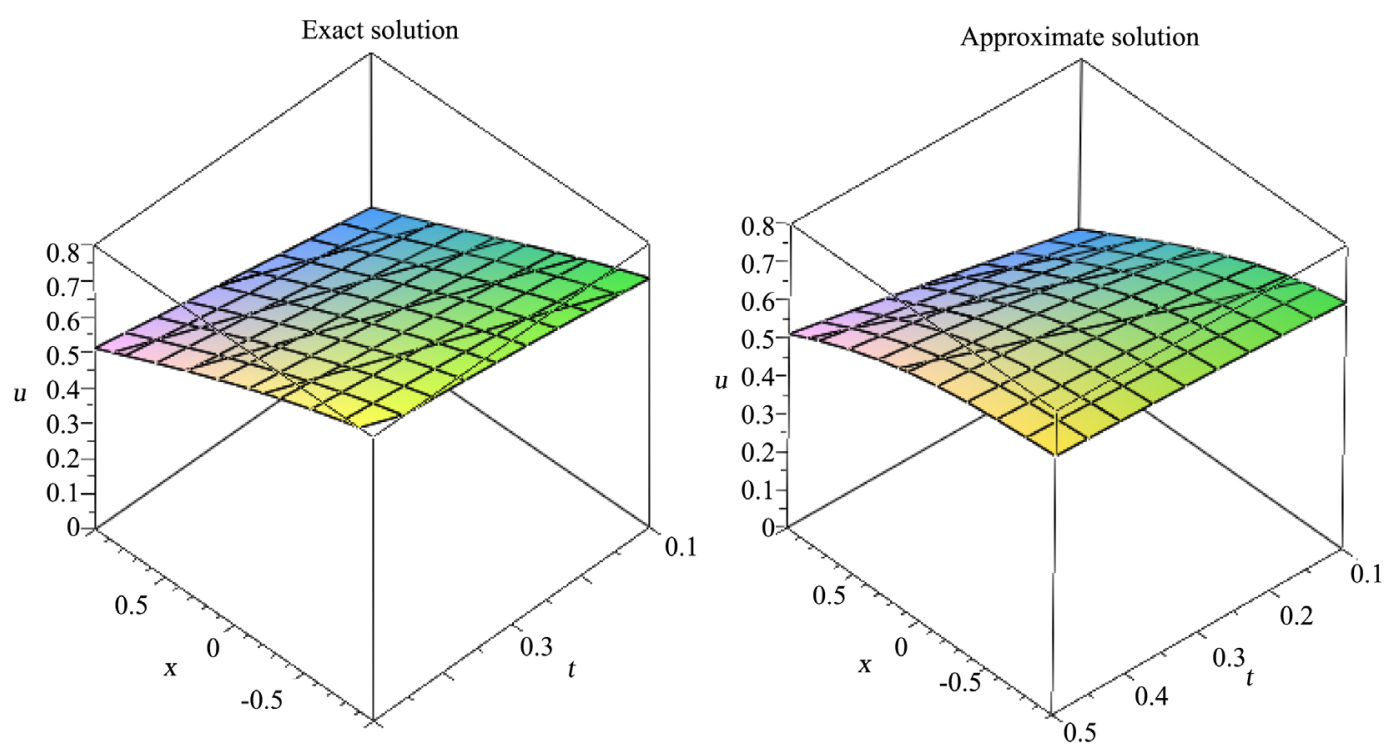

Figure 2. Exact and approximate solutions to Cahn-Hilliard equation by ADM when $-1 \prec x \prec 1$ and $t=0.1,0.2,0.3,0.4,0.5$.

references [18], a correction functional for Equation (43) can be written as

$$
L u+N u=N u_{n+1}(t)=u_{n}(t)+\int_{0}^{t} \lambda\left(L u_{n}(s)+N u_{n}(s)-f(s)\right) \mathrm{d} s
$$

where $\lambda$ is a general Lagrange's multiplier [17], which can be identified optimally via the variational theory, and $\tilde{u}_{n}$ is are stricted variation which means $\delta \tilde{u}_{n}=0$. The successive approximations $u_{n+1}, n \succ 0$, of the solution $u$ will be readily obtained upon using the determined Lagrangian multiplier and any selective function $u_{0}$. Therefore, the solution is given by

$$
u=\lim _{n \rightarrow \infty} u_{n}
$$

\section{Application of VIM}

Considering the BBM Equation $(18,19,20)$

$$
u_{t}=u_{x x t}-u_{x}-u u_{x},
$$

The VIM can be applied to Equation (18) in the form

$$
u_{n+1}(x, t)=u_{n}(x, t)+\int_{0}^{t} \lambda\left(\left(u_{n}\right)_{t}(x, s)+\left(u_{n}\right)_{x}(x, s)+\left(u_{n}\right)\left(u_{n}\right)_{x}(x, s)-\left(u_{n}\right)_{x x t}(x, s)\right) \mathrm{d} s
$$

where $\lambda$ yields to -1 . One can substitute above $\lambda$ and use the initial approximation as in Equation (45) to get the successive approximation by the following expressions:

$$
\begin{gathered}
u_{0}=\operatorname{sech}^{2}(x / 4) \\
u_{1}(x, t)=u_{0}(x, t)+\int_{0}^{t} \lambda\left(\left(u_{0}\right)_{t}+\left(u_{n}\right)_{x}+\left(u_{n}\right)\left(u_{0}\right)_{x}-\left(u_{0}\right)_{x x t}\right) \mathrm{d} s \\
u_{1}(x, t)=\operatorname{sech}^{2}\left(\frac{x}{4}\right)+\frac{t}{2}\left\{\operatorname{sech}^{2}\left(\frac{x}{4}\right) \cdot \tanh \left(\frac{x}{4}\right)+\operatorname{sech}^{4}\left(\frac{x}{4}\right) \cdot \tanh \left(\frac{x}{4}\right)\right\} \\
u_{2}(x, t)=u_{1}(x, t)+\int_{0}^{t} \lambda\left(\left(u_{1}\right)_{t}+\left(u_{1}\right)_{x}+\left(u_{1}\right)\left(u_{1}\right)_{x}-\left(u_{1}\right)_{x x t}\right) \mathrm{d} s
\end{gathered}
$$


The Cahn-Hilliard equation:

$$
u_{t}=u_{x x}-u^{3}+u \text {, }
$$

the VIM can be applied to Equation (30) in the form

$$
u_{n+1}(x, t)=u_{n}(x, t)+\int_{0}^{t} \lambda\left(\left(u_{n}\right)_{t}-\left(u_{n}\right)_{x x}+\left(u_{n}\right)^{3}-\left(u_{n}\right)\right) \mathrm{d} s
$$

where $\lambda$ yields to -1 . One can substitute above $\lambda$ and use the initial approximation as in Equation (50) to get the successive approximation by the following expressions:

$$
\begin{aligned}
& u_{0}=\frac{1}{1+\mathrm{e}^{\frac{x}{\sqrt{2}}},} \\
& u_{1}(x, t)=u_{0}(x, t)+\int_{0}^{t} \lambda\left(\left(u_{0}\right)_{t}-\left(u_{0}\right)_{x x}+\left(u_{0}\right)^{3}-\left(u_{0}\right)\right) \mathrm{d} s \\
& u_{1}(x, t)=\frac{1}{1+\mathrm{e}^{x / \sqrt{2}}}-\int_{0}^{t}\left\{-\left[\frac{\mathrm{e}^{2 x / \sqrt{2}}-\frac{1}{2} \mathrm{e}^{x / \sqrt{2}}\left(1+\mathrm{e}^{x / \sqrt{2}}\right)}{\left(1+\mathrm{e}^{x / \sqrt{2}}\right)^{3}}\right]+\left(\frac{1}{1+\mathrm{e}^{x / \sqrt{2}}}\right)^{3}-\left(\frac{1}{1+\mathrm{e}^{x / \sqrt{2}}}\right)\right\} \mathrm{d} s \\
& u_{1}(x, t)=\frac{1}{1+\mathrm{e}^{x / \sqrt{2}}}+\frac{3 / 2}{\left(1+\mathrm{e}^{x / \sqrt{2}}\right)^{2}}(t) \\
& u_{2}(x, t)=u_{1}(x, t)+\int_{0}^{t} \lambda\left(\left(u_{1}\right)_{t}-\left(u_{1}\right)_{x x}+\left(u_{1}\right)^{3}-\left(u_{1}\right)\right) \mathrm{d} s
\end{aligned}
$$

\section{Conclusion}

In this work, the Adomian Decomposition Method and the Variational Iteration Method have been successfully applied to find the solution of nonlinear Benjamin-Bona-Mahony and Cahn-Hilliard equations are presented in Figures 1-4. It was observed that the use of ADM and VIM provides a very good estimation when compared
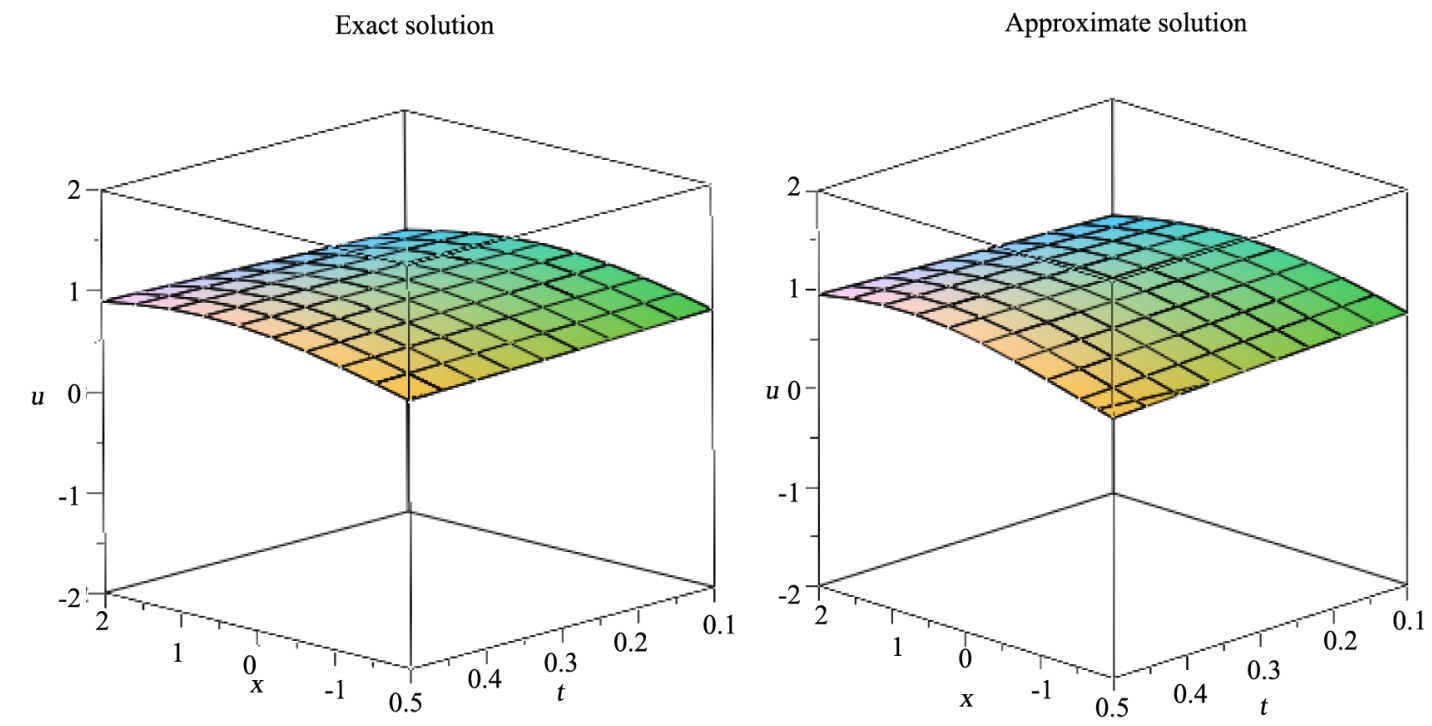

Figure 3. Exact and approximate solutions to BBM equation by VIM when $-2 \prec x \prec 2$ and $t=0.1,0.2,0.3,0.4,0.5$. 

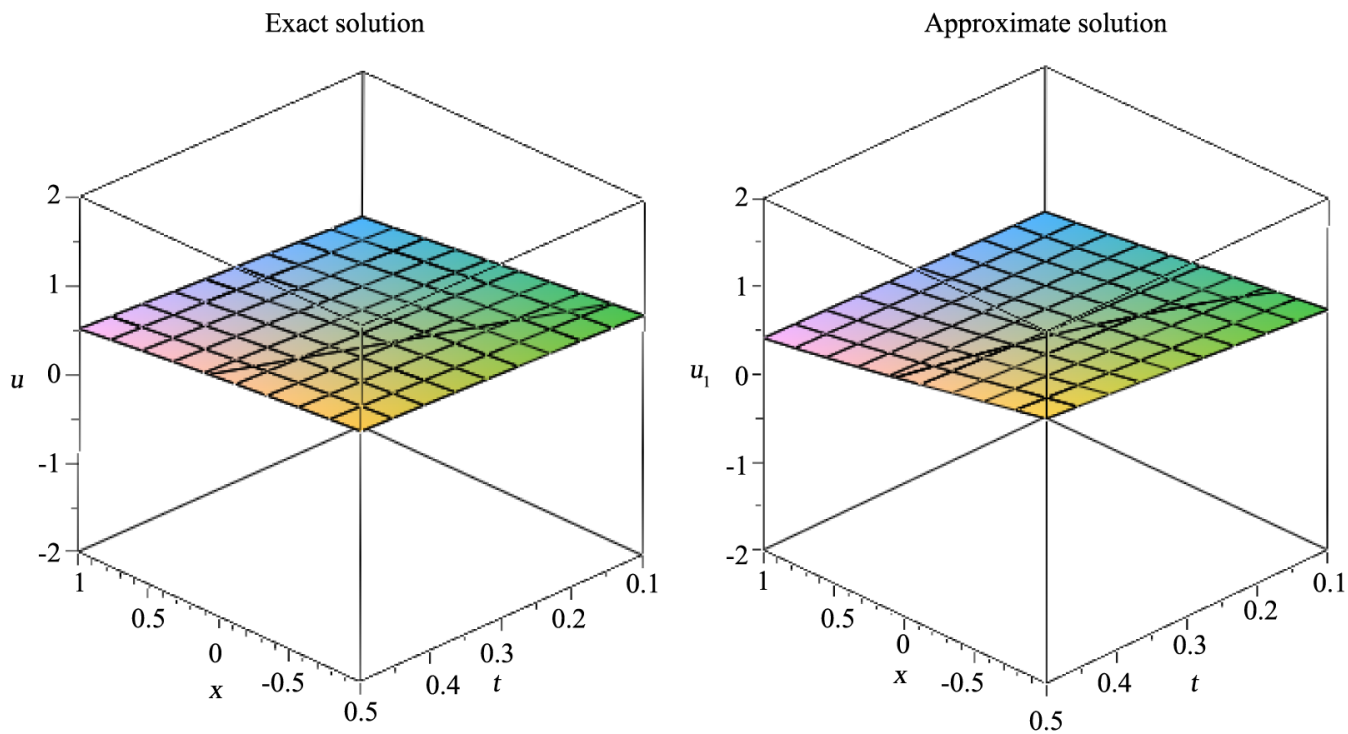

Figure 4. Exact and approximate solutions to Cahn-Hilliard equation by VIM when $-1 \prec x \prec 1$ and $t=0.1,0.2,0.3,0.4,0.5$.

with exact values. These methods convert these equations to recurrences relation whose terms are computed using maple 15. This method is very effective and accelerates the convergent of solution.

\section{References}

[1] Benjamin, T.B., Bona, J.L. and Mahony, J.J. (1972) Model Equations for Long Waves in Nonlinear Dispersive System. Philosophical Transactions of the Royal Society A, 272, 47-78.

[2] Ugurlu, Y. and Kaya D. (2008) Solutions of the Cahn-Hilliard Equation. Computers and Mathematics with Applications, 56, 3038-3045. http://dx.doi.org/10.1016/j.camwa.2008.07.007

[3] Wazwaz, A.-M. (2005a) Exact Solutions of Compact and Noncompact Structures for the KP-BBM Equation. Applied Mathematics and Computation, 169, 700-712. http://dx.doi.org/10.1016/j.amc.2004.09.061

[4] Wazwaz, A.-M. (2005b) Compact and Noncompact Physical Structures for the ZK-BBM Equation. Applied Mathematics and Computation, 169, 713-725. http://dx.doi.org/10.1016/j.amc.2004.09.062

[5] Wazwaz, A.-M. (2005c) Exact Solutions with Compact and Noncompact Structures for the One-Dimensional Generalized Benjamin Bona Mahony Equation. Communications in Nonlinear Science and Numerical Simulation, 10, 855867. http://dx.doi.org/10.1016/j.cnsns.2004.06.002

[6] Al-Khaled, K., Momani, S. and Alawneh, A. (2005) Approximate Wave Solutions for Generalized Benjamin Bona Mahony Burgers Equations. Applied Mathematics and Computation, 171, 281-292. http://dx.doi.org/10.1016/j.amc.2005.01.056

[7] Furihata, D. (2001) A Stable and Conservative Finite Difference Scheme for the Cahn-Hilliard Equation. Numerische Mathematik, 87, 675-699. http://dx.doi.org/10.1007/PL00005429

[8] Kim, J. (2007) A Numerical Method for the Cahn-Hilliard Equation with a Variable Mobility. Communications in Nonlinear Sciences and Numerical Simulation, 12, 1560-1571. http://dx.doi.org/10.1016/j.cnsns.2006.02.010

[9] Wells, G.N., Kuhl, E. and Garikipati, K. (2006) A Discontinuous Galerkin Method for the Cahn-Hilliard Equation. Journal of Computational Physics, 218, 860-877. http://dx.doi.org/10.1016/j.jcp.2006.03.010

[10] Kourosh, P. and Jamal, A.R. (2012) Exp-Function Method for Some Nonlinear PDE's and a Nonlinear ODE's. Journal of King Saud University, 24, 1-10. http://dx.doi.org/10.1016/j.jksus.2010.08.004

[11] Alquran, M.T. (2012) Applying Differential Transform Method to Nonlinear Partial Differential Equations: A Modified Approach. Applications and Applied Mathematics, 7, 155-163.

[12] Adomian, G., Rach, R. and Shawagfeh, N.T. (1995) On the Analytic Solution of the Lane-Emden Equation. Physics Letters, 8, 161-181. http://dx.doi.org/10.1007/bf02187585

[13] Adomian, G. and Serrano, S.E. (1998) Stochastic Contaminant Transport Equation in Porous Media. Applications and Applied Mathematics, 11, 53-55. http://dx.doi.org/10.1016/S0893-9659(97)00132-8 
[14] Adomian, G. (1994) Solving Frontier Problems of Physics: The Decomposition Method. Kluwer Academic Publishers, Boston.

[15] Mohamed, M.S. (2015) Adomian Decomposition Method for Solving Coupled KdV Equations. Journal of Advances in Mathematics, 10, 3472-3478.

[16] Abdou, M.A. (2005) Adomian Decomposition Method for Solving the Telegraph Equation in Charged Particle Transport. Journal of Quantitative Spectroscopy and Radiative Transfer, 95, 407-414. http://dx.doi.org/10.1016/j.jqsrt.2004.08.045

[17] Wu, G.C. (2013) Challenge in the Variational Iteration Method-A New Approach to Identification of the Lagrange Multipliers. Journal of King Saud University—Science, 25, 175-178. http://dx.doi.org/10.1016/j.jksus.2012.12.002

[18] He, J.H. (1999) Variational Iteration Method-A Kind of Non-Linear Analytical Technique: Some Examples. International Journal of Non-Linear Mechanics, 34, 699-708. http://dx.doi.org/10.1016/S0020-7462(98)00048-1 